
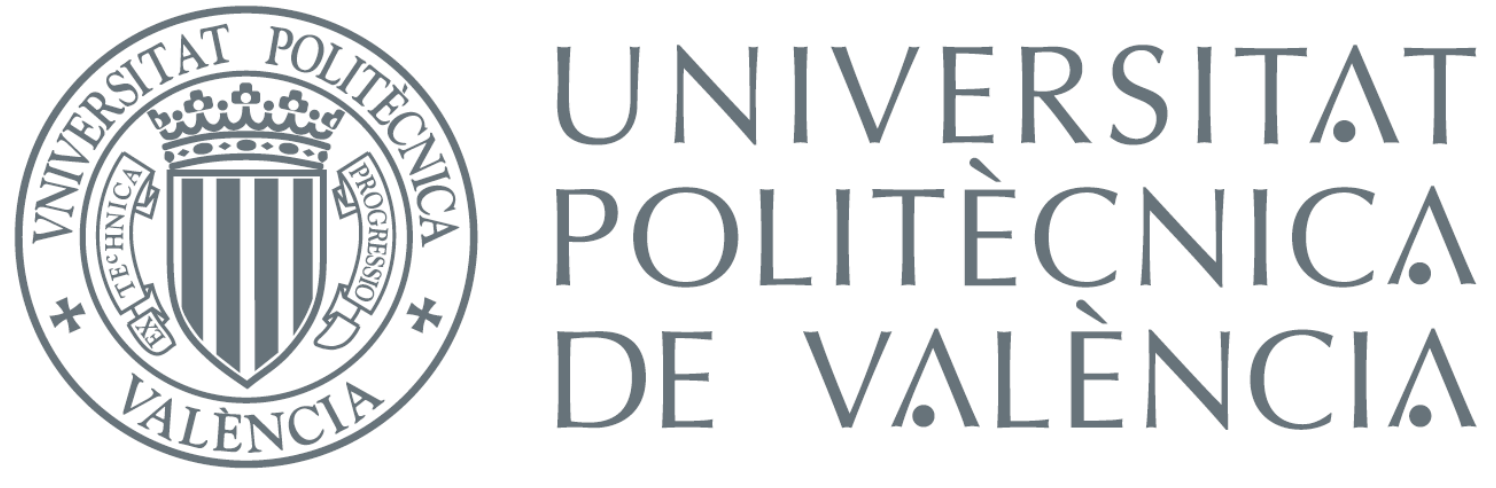

Departamento de Ingeniería Electrónica

\title{
Sound Scattering by Lattices of Heated Wires.
}

$\underline{\text { Por Mitko Ivanov Angelov }}$

Dirigida por

Francisco Cervera Moreno

$$
\mathrm{y}
$$

José Sánchez-Dehesa Moreno-Cid 


\section{Contents}

Outline of the PhD manuscript and Methodological strategy............1

I Focusing Effect of Two Hot Wires.............................................3

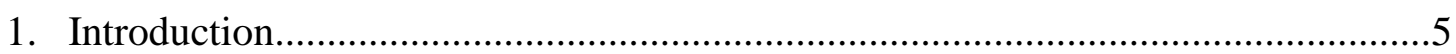

1.1. Temperature gradient as a natural phenomenon..............................................

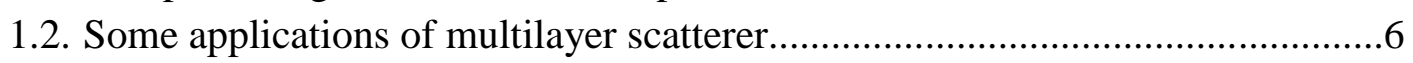

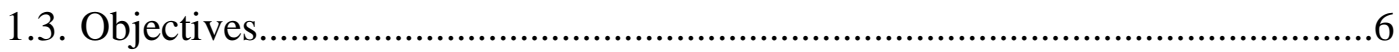

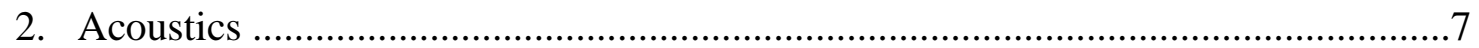

2.1. Acoustic wave equation............................................................................

2.2. Ultrasound in real and ideal gases...............................................................

2.3. Reflection and transmission of acoustic waves................................................

2.4. Transmission through a fluid layer: normal incidence.....................................10

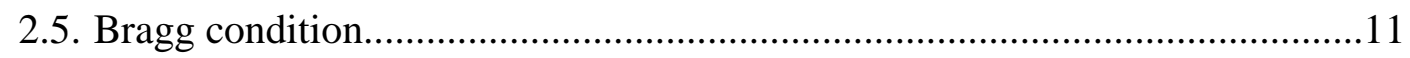

3. Fundamentals of the heat transfer theory............................................................13

3.1. The heat equation.......................................................................................13

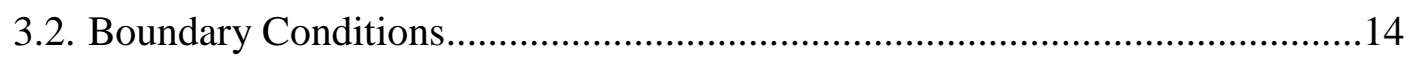

4. Focusing of ultrasonic waves by an array of hot wires..........................................17

5. Experimental confirmation of the focusing effect produced by heated wires..........23

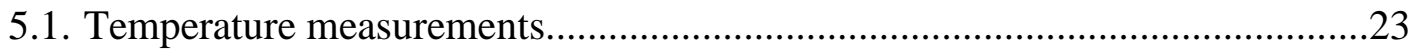

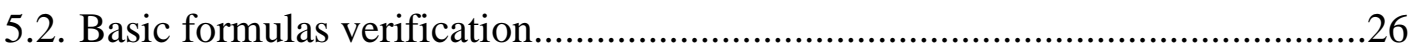

5.3. Temperature and pressure measurements near the heated wire.........................29

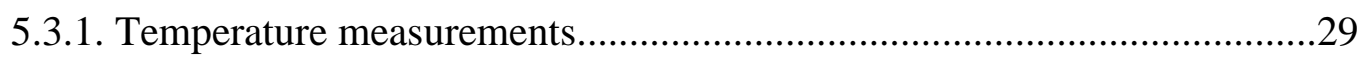

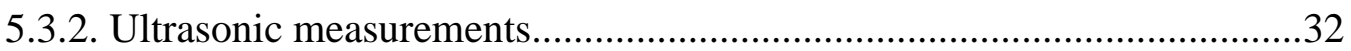

5.4. Comparison between theory and experiments …………………………........40

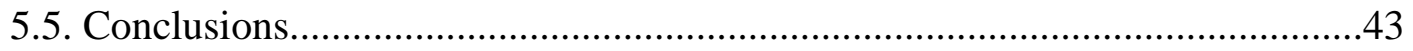

II Sonic Crystals Made of Heated Wires....................................................45

6. Introduction to Sonic Crystals............................................................................4

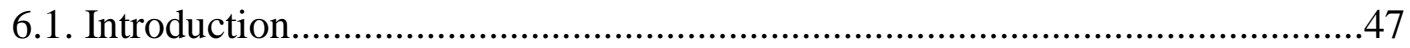

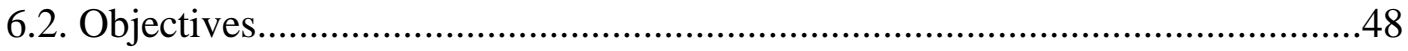

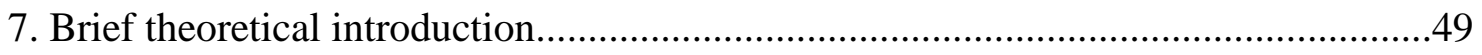

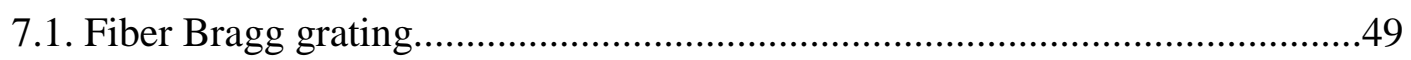

7.2. Standing wave and acoustic interferometers..................................................

8. Two-dimensional model of sonic crystal. Numerical simulations..................................53

8.1. Crystal definition......................................................................................

8.2. Temperature field inside the sonic crystal.......................................................

8.3. Acoustic pressure simulations.....................................................................64

8.4. Acoustic analogue of Fiber Bragg grating....................................................66 
8.5. Reflection coefficients as a function of the temperature..................................68

9. Experimental study of the properties of SC made of heated wires............................71

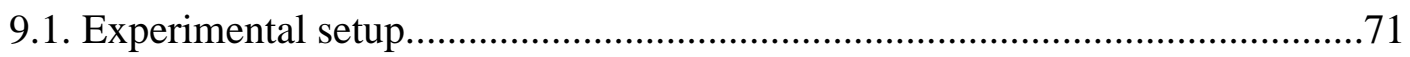

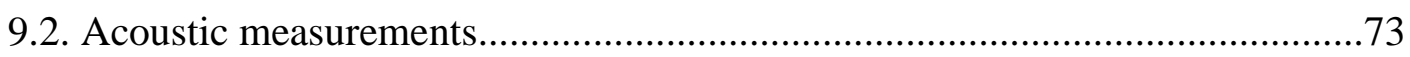

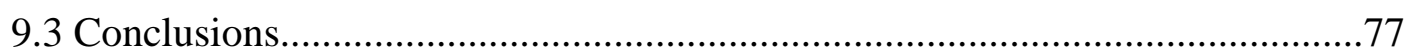

III Gradient-Index Sonic Crystals.........................................................79

10. Gradient-Index Sonic Crystals - introduction and objectives..................................81

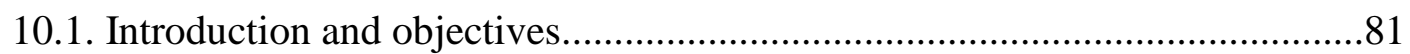

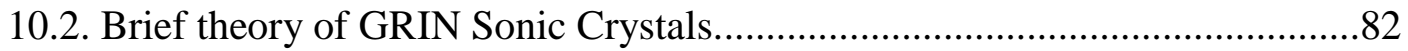

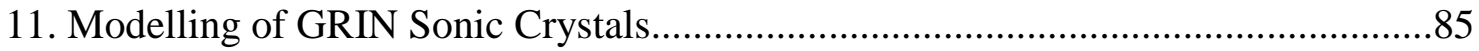

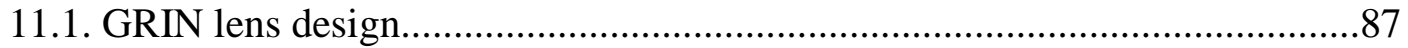

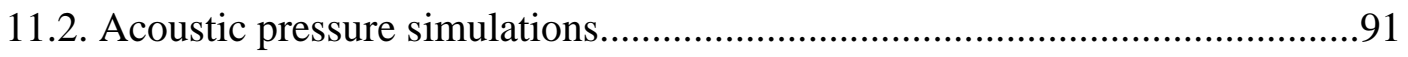

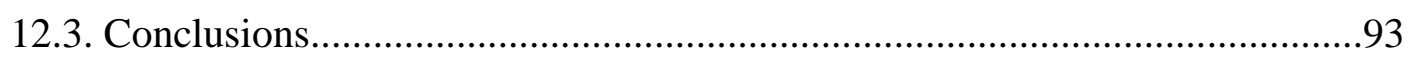

Concluding remarks.............................................................................95

Appendices....................................................................................101

A Acoustic Wave Equations Derivation...........................................103

B Theory of Multilayered Scatterer.....................................................109

C Heat Transfer Equations...........................................................117

D The Finite Element Method...............................................................131

E Publications.............................................................................................157

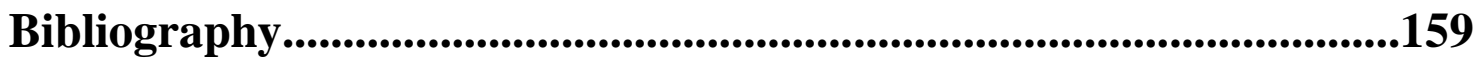

